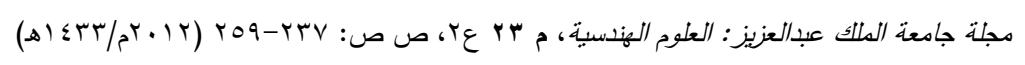

\title{
تتبع الحركة القلبية في صور الرنين المغنطيسي باستخدام طريقة تسجيل الصور اللاخطي
}

\author{
رنـا حدّاد \\ قسم الهندسة الطبية، كلية الهندسة المبكانيكية والكهرائية، جامعة دمشق، سوربيا \\ haddadrana@yahoo.fr
}

المستخلص. إن عملية تتبع حركة القلب تساعد الطبيب على تحديد

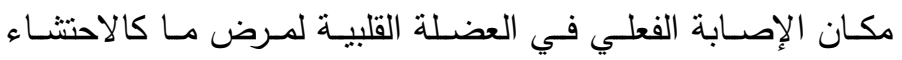

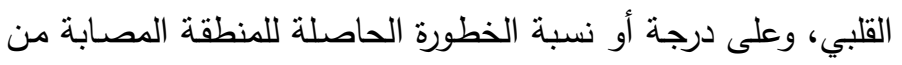

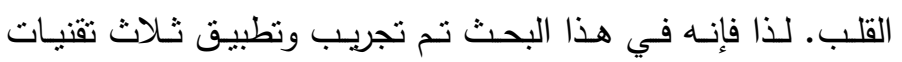

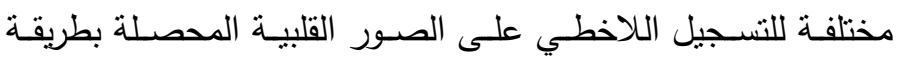

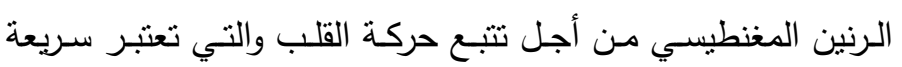
وذات سعة صغيرة. أولى هذه التقنيات بعتمد على تطبيق خوارزمية

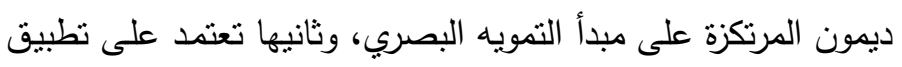

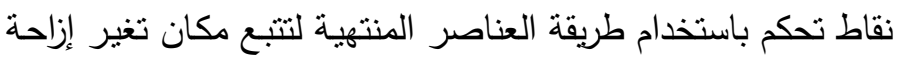

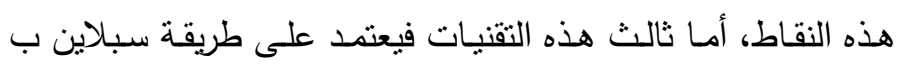

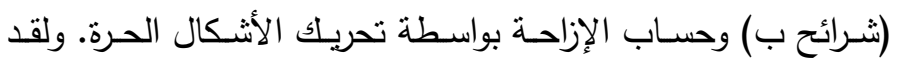

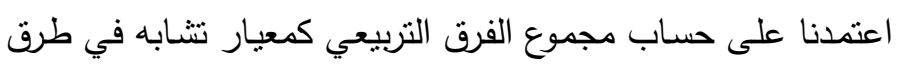

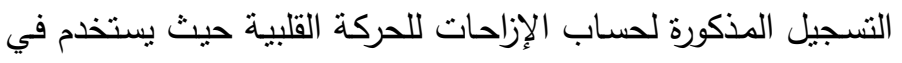

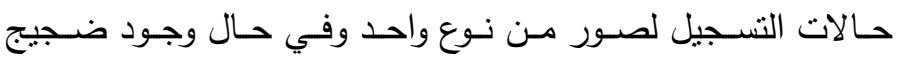




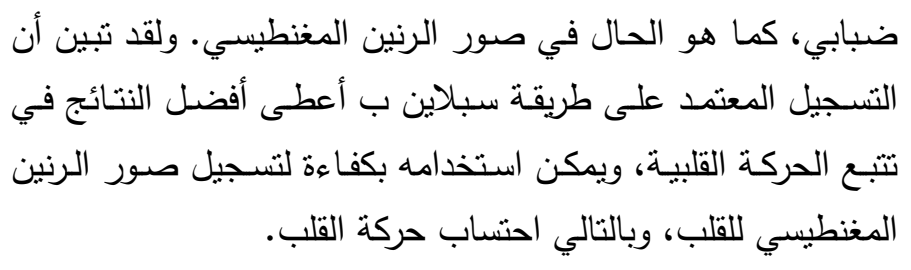

الكلمات المفتاحية: صور الرنين المغنطيسي، تسجيل الصور الطبية، طرق التسجيل اللاخطية، تتبع حركة القلب.

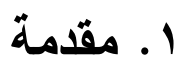

يعتبر القلب من أهم أعضـاء الجسم ويعبّر عن حيويـة الكائن الحيّ وإن سـرعة الكثف عن إصـابة هذا العضو أو جزء منـه بمرض مـا يؤدي إلى تفادي الأذيـة القليبة أو حتى من الجلطات الدموية والتي بدورها قد تؤدي إلى الموت المفاجئ. أدى تطور العلم والتقنيات بشكل ملحوظ في الآونـة الأخيرة إلى توفير المسـاعدة

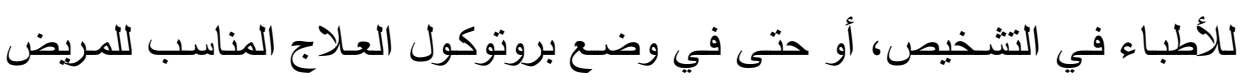

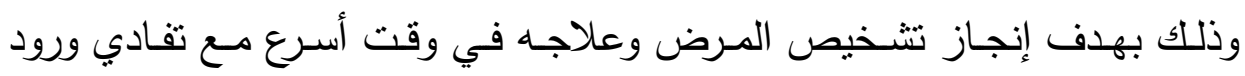
الخطأ في العـلاج المقترح وهذا كله أدى إلى أنمتـة أسـاليب التشـخيص وطـرق

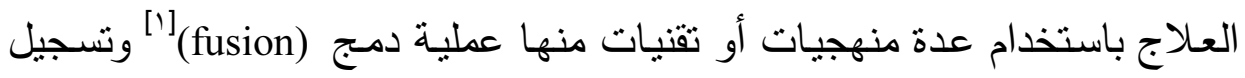

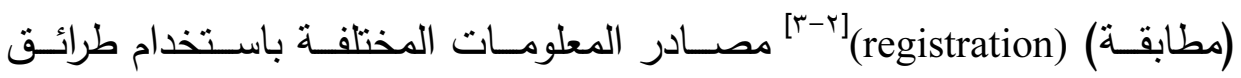
التصـوير الطبيـة المتتوعـة مثل: الرنين المغنطيسي ( Magnetic Resonance (Images (MRI) الرنين المغنطيسي المعلّم Tagged MRI... حيث يتطلب عملية تسجيل المعلومات خطوة تحويـل بدائيسة بهدف تعـويض الاختلاف الهندسـي أو الاختلاف في شـدة الكثافة بين مجموعات البيانـات، وهو العنصـر الرئيسي في تنسجيل الصـور (أو المطابقة). هذا الأخير أصبح واحداً من أكثر التقنيات استخداماً في مجال الروئية بالحاسب حيث تتضمن تطبيقاته التدفق البصري (optical flow)، وتحليل الحركة 


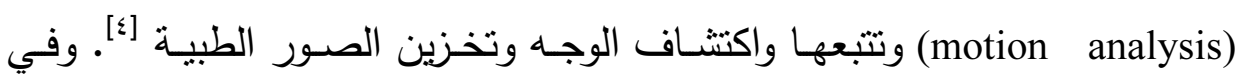
بعض الحالات يكون التسجيل الخطي (linear) الممثل بالتحويلات الصلبة (rigid) والتآلفيـة (affine) كافياً، وفي الحالات الأكثر تعقيداً، منثل تحليل حركة أعضـاء الجسـم، سـوف يـتم اللجـوء إلـى طريقـة أعقد وهـي طريقـة التســيل اللاخطيـة والتي تكون مهمة ومفيدة في رصد الحركات المركبة مثل حركة القلب (nonlinear) لكونسه عضواً لله تعقيده من حيث البنية والتراكيب التشريحية (cardiac motion) المؤلفـة لـه، وللتداخل الكبير بين هذه التراكيب وخصوصـاً في حركتهـا المعقدّة

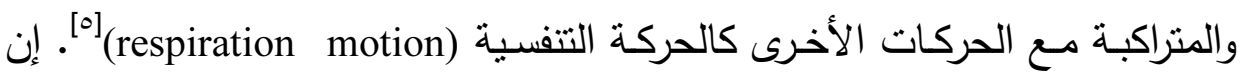
التقنيات المقترحة في مجال تتبع حركة القلب وحساب الإزاحات (displacements) الشـاملة لحركة القلب بطريقة التسـيل أصبحت أكثر تطرقاً ودراسـةً نظراً للنتائج المقنعـة التي تعطيها بالمقارنـة مـع الطرق السـابقة كطرق الانسياب العـائم [־]، أو باستخدام نموذج بدائي بهدف تتبع حواف الأجزاء القلبية وبشكل خاص البطين

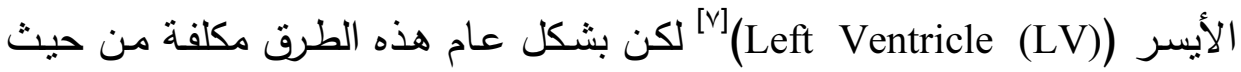
الوقت وتحتاج لمعرفة أولية بشكل حواف (كفاف) الأجزاء القلبية (contours). لذا هال فالهدف مـن هذا العمل هو دراسـة طرق التسـيل اللاخطيـة وتقييمها في إطـار تطبيقها على تصوير القلب التشريحي (anatomical) والديناميكي (dynamical) بالرنين المغنطيسي نظراً لأهمية هذا العضو -القلب- وتتبع حركته، وللاستخدام الواسع والثامل للكثف عن أمراض القلب باستخدام هذه التقنية (MRI)، حيث في

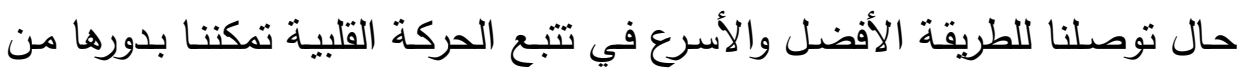
الحصول على دلائل عن درجة ومكان توضع الضرر والأذى في العضلة القلبية، وبالتالي تساعد في عملية أتمتة وسرعة الكثف والتشخيص ومل لمرض قلبي ما. إن الطرق المستخدمة في تتبع الحركة القلبية والمعتمدة على طريقة التسجيل

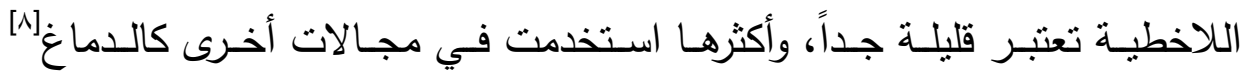
والصـدر (أورام الثدي)]•(،9] وتم تطبيقها على أنـواع أخـرى مـن التصـوير القلبي 


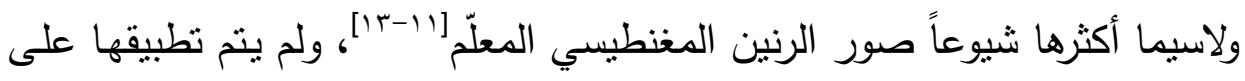
القلب باستخدام صور الرنين المغنطيسي العادي. وبما أن الحصول على هذا النوع

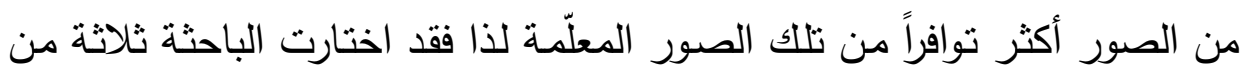
الطرق الأكثر شيوعاً في الاستخدام في التسجيل اللاخطي لتتبع الحركة الصغيرة المطال والسريعة كحركة القلب، لتطبيقها وتجربتها على صور الرنين المغنطيسي التي للقلب لكون حركته تتمثل في أقل من ثانية واحدة في الدورة القلبية ( cardiac cycle (CC) من أجل المقارنة بين هذه الطرق وإعطاء دلائل لأفضلية استخدام طريقة

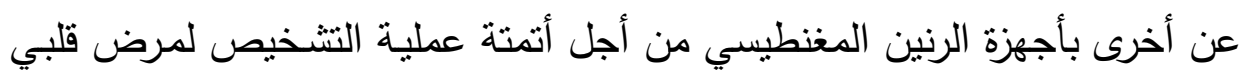
ما ولاسيما الإحتشاء القلبي (ischemia) عند استخدام تقنية الرنين المغنطيسي في

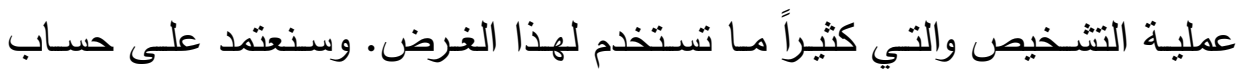
مجموع الفرق التربيعي كمعيار نتابه في طرق التسجيل المذكورة لحساب الإزاحات

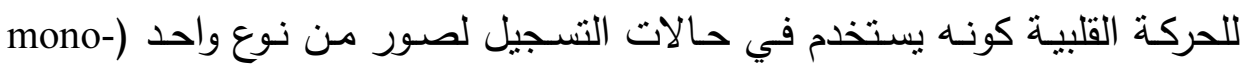
(modality بدقة أكبر في حال استخدام التسجيل في مطابقة الصور من مصسادر معلومات مختلفة (multi-modality) ويستخدم هذا المعيار (مجموع الفرق التربيعي) أيضاً في مي مئري حال وجود ضجيج ضبابي كما هو الحال في صور الرنين المغنطيسي المستخدمة في هذه الدراسة.

r Medical Image Registration طريقة تسجيل الصور الطبية

r - r

طريقة تسجيل الصور هي إحدى أهم طرق معالجة الصور الرقية [؛]؛ حيث

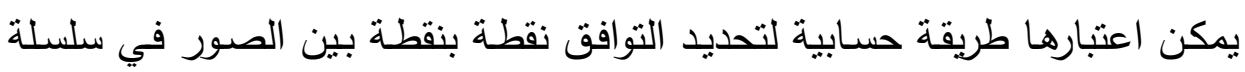
ما، أي تحليل التطور الزمني (الحركة)، أو قد تستخدم كمصدر معلومات إضـافية 
عـن الصـور لتقـدير الفـروق بـين الثـكل الهندسـي و /أو كثافـة الصـور أو قيم

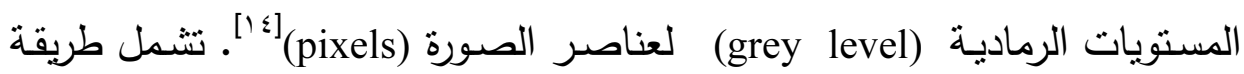
التسجيل على إيجاد دالة تحويل (transform function) رياضي لتعويض التغيرات

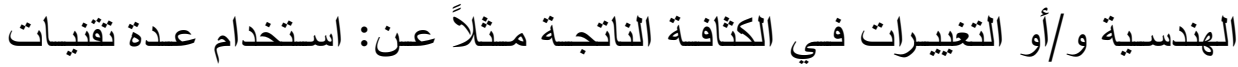

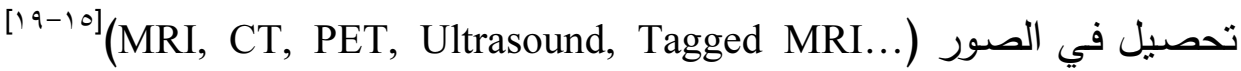
لدمج الصور أو الاختلاف في شروط، أو هندسة تحصيل الصور ، أو في استخدام

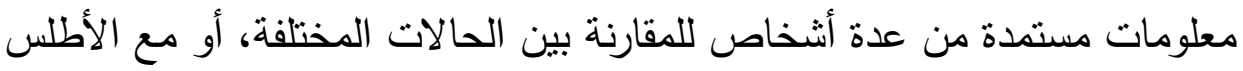

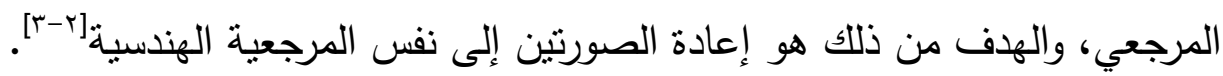
وتتمثل عمليـة التسـيل في تحديد دالـة تحويل T الذي يتعلق بإحداثيات عناصر صورة A المسماة الصورة العائمة (floating) (التي يطبق عادةً عليها دالة التحويل) وعناصر الصورة B التي تسمى الصورة المرجعية (reference) ومن ثم تحقيق المستوى الأمثل لمعيار قياس النتـابه (similarity metric) على المعلومات المستمدة من هذه البيانات.

يعرف الفضاء العام الذي يحوي فضائي الصورتين بالعلاقة (1):

$$
\Omega_{A, B}^{T}=\left\{A\left(x_{A}\right) \in \Omega_{A} / T\left(x_{A}\right) \in \Omega_{B}\right\}: A, B
$$

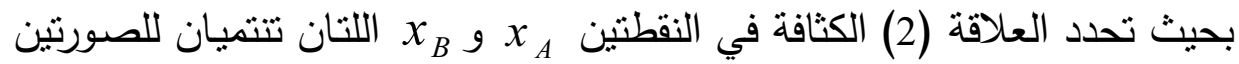
A و B على التنالي:

$$
\begin{aligned}
& A: x_{A} \in \Omega_{A} \mapsto A\left(x_{A}\right) \\
& B: x_{B} \in \Omega_{B} \mapsto B\left(x_{B}\right)
\end{aligned}
$$

فإن عملية تسجيل الصورة A مع الصورة B بتطبيق دالة التحويل T تعرف بالعلاقة

$$
x_{A} \in \Omega_{A} \mapsto T\left(x_{A}\right)=x_{B} \in \Omega_{B}
$$


وبيين الشكل ا المبدأ العام لعملية التسجيل بين الصورتين A، B:

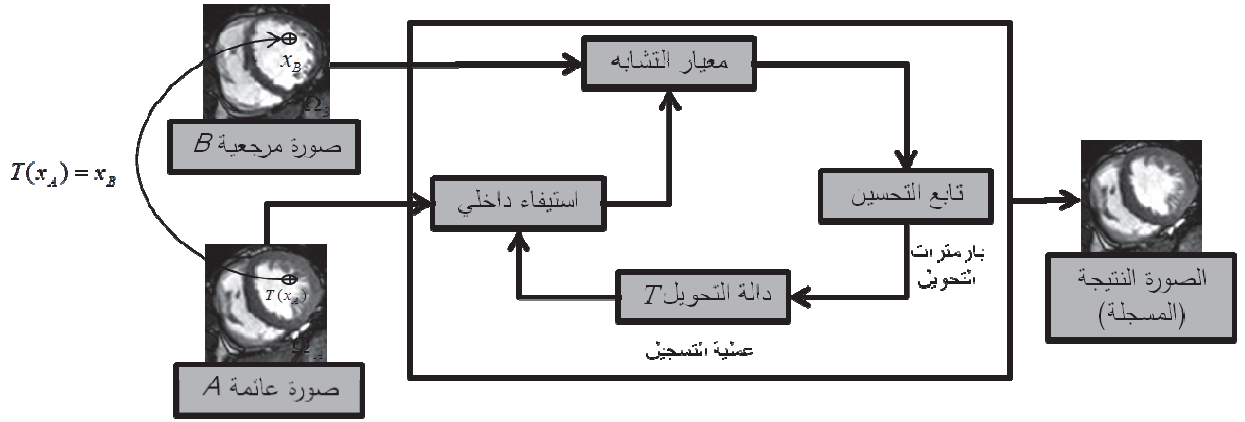

شكل 1ـ المبدأ العام لعملية التسجيل بين الصورة المرجعية B والصورة العائمة A.

وتوجد عدة طرق لحساب معيار التشابه بين الصور والذي هو عبارة عن

معيار يسمح بتقييم نوعية طريقة التسجيل المستخدمة ويحسب في المجال العام للصورتين sum of ) في شدة الكثافة بين الصورتين. من هذه الطرق: مجموع الفرق المطلق (absolute difference)، ومجموع الفرق التربيعي (sum of squared difference) اللذان يستخدمان في حال قياس تطابق الصور من نوع واحد مثل (MRI) فقط أو [9 "19]، أما معامل الارتباط (Tagged MRI)

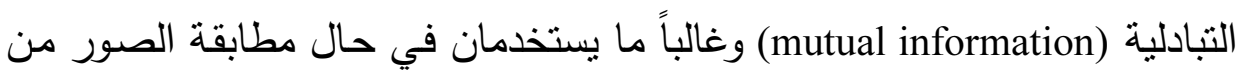

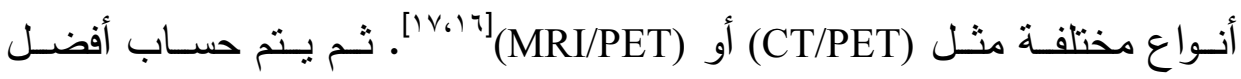

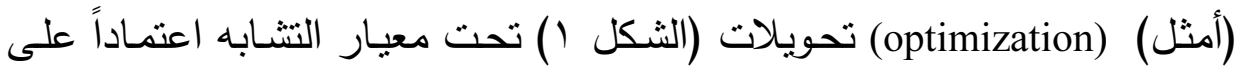
الافتراضـات والقيود الموضـوعة في بدايـة تسـيل الصـور ، ويتم إمـا حلها تحليليًا بايجاد الخطأ الأصغر للقيم التربيعية (minimize the mean-squared error) أو قد تتطلب طرقاً تكرارية كاستخدام طريقة الانحدار المتدرج (gradient descent). وهنـالك عدة تقنيات لتسـيل الصسور تعود إلى نوع التغييرات في الصسور ومجال الدراسة. وتقسم عموماً إلى خطية ولا خطية. 
الخطبة Linear: تستخدم عادةً في الأنظمة الخطية وغالباً ما تُشتخدم بهدف تبسيط عملية التسجيل وهي إما تحويلات صلبة و/أو تحويلات تآلفية. والتحويلات الصلبة تؤدي إلى تغييرات شاملة في الصور منل التحويلات الهندسية (الشكل r): انسحاب، تقييس، تحجيم (تصغير) و /أو دوران، أما التحويلات التآلفية وتأخذ بعين الاعتبار العديد من التشوهات المكانية المعتادة.

أسلوب التسجيل غير الصلب أو المسن (اللاخطي) NonLinear: تستخدم

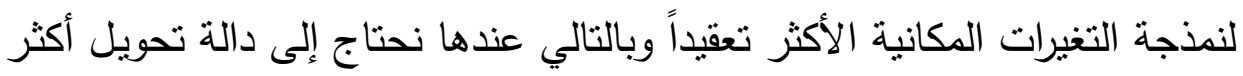
تعقيداً لكي يعطي نتائج أدق والمزيد من درجات الحرية (الشكل ب). يتم استخدامها

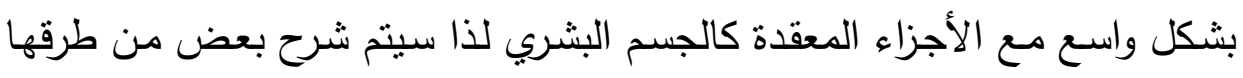
بشكل موسع لأنه ينصب في مجال هذا البحث.
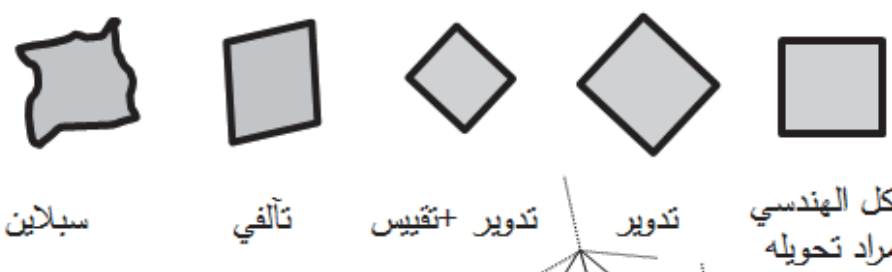

لاخطي باستخدام شبكة

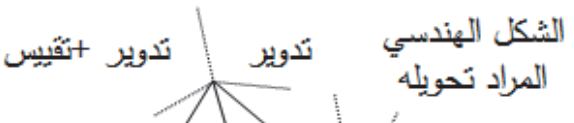

شكل r ـ بعض نتائج التحويلات الهندية الصلبة واللاخطية على شكل هندي (مريع). r هناك العديد من خوارزميات التسجيل المرنة المقترحة في الدراسات المرجعية ويوجد العديد من دراسات المقارنة التي أُجريت في مجال التصوير الدماغي ونطاق 
الصدر باستخدام صور الماموغرام[9] على سبيل المثال. لكن لا يوجد إلا عدد قلبل من الخوارزميات التي جرى اختبارهـا مـع صسور القلب ولا سيما باستخدام صـور

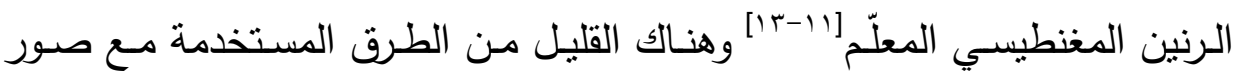

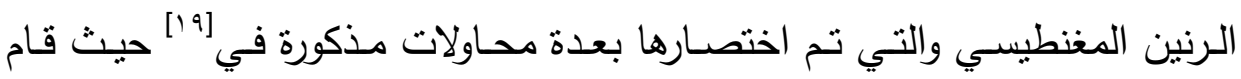
ماكيلا ورفاقه بدراسـة مرجعيـة شـاملة لمختلف طرق التسجيل سواءً بهدف تطابق الصور القلبية من أنواع تصوير مختلفة أو بهدف تتبع الحركة، نذكر منهم: لوتتين ورفاقـهـ[r] الـذين طبقـوا التسـجيل غيـر الصـلب (المـرن) معتمـدين على تحريـك الأشكال الهندسية المشابهة لشكل القلب لتطابق الصور القلبية بمختلف أنواعها. وهناك أيضاً دولكريك ورفاقه[rا] الذين اقترحوا أسلوب تسجيل غير خطي للصور القلبية بهدف احتساب حركة القلب. إن أغلب الدراسات تعتمد على استخدام صور

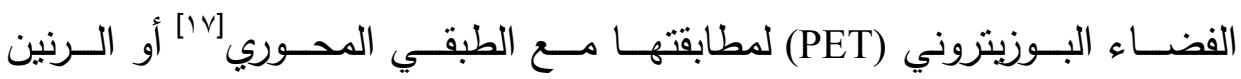
المغنطيسي [1 1]، فالقليل جداً من الدراسـات التي استخدمت طريقة تسـيل الصور

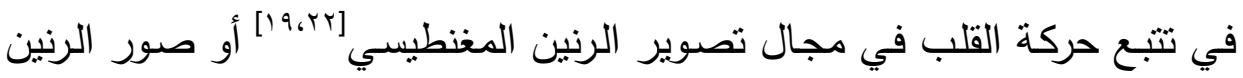

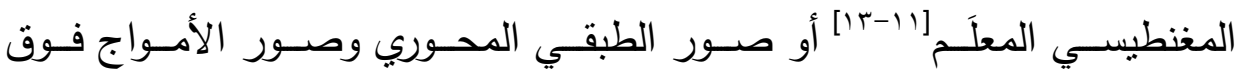

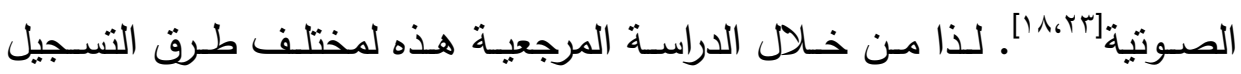
اللاخطية ارتأت الباحثة دراسـة عدد من الطرق وتطبيقها في مجال تتبع حركة هـن

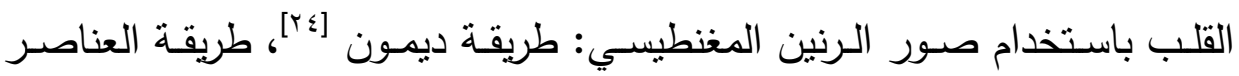

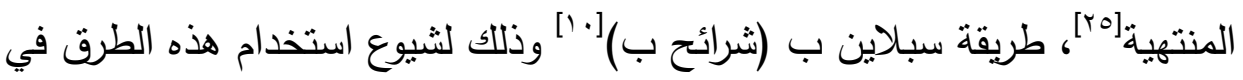
تتبع الحركات ذات السرعة الكبيرة والمطال الصغير أي من رثبة حركة القلب. أ- طريقة ديمون Demons Algorithm

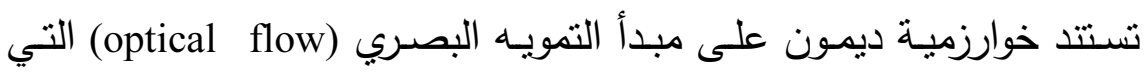
تسـمح بتقدير حركات صـغيرة في سلاسـل الصـور الديناميكيـة، حيـث يعتبر كل صورة كمجموعة من الكفاف (contours) ذو كثافة متتاغمة (iso-intensity) حيث 
$r \leqslant 0$

يتم تحريكهم بواسطة شبكة منتظمة من القوى وذلك عن طربق قوة دفع الكفاف p باتجاه الناظم (normal) بطربقة تكرارية كما في الثكل س وباعتبار I تمثل الكثافة عند نقطة من الصورة و v تمنثل السرعة عندها بالعلاقة (4): $\vec{v}=\frac{-I_{x} \vec{I}_{t}}{\left|\overrightarrow{I_{x}}\right|^{2}+I_{t}^{2}}$

$$
\text { حيث تعرف }
$$

$$
I_{t}=\frac{\partial I}{\partial t}, \vec{I}_{x}=\left(\frac{\partial I}{\partial x}, \frac{\partial I}{\partial y}\right)^{T}
$$

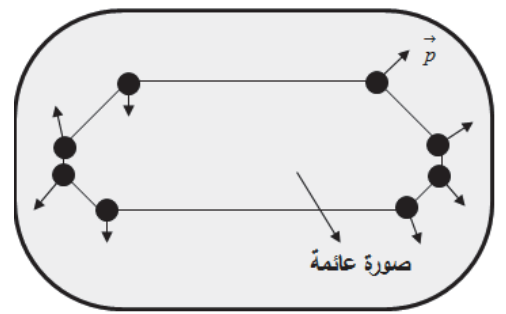

شكل r. شبكة ديمون مطبقة على الصورة العائمة.

فإنه بعبر عن مطال واتجاه الإزاحة باستخدام منطق التموبه البصري للإزاحات الصغيرة بالعلاقة (6):

$$
\vec{p} \cdot \vec{\nabla} b=m-b
$$

وبالتالي يعبر عن قوة دفع الكفاف p باستخدام علاقة السرعة (4) بالعلاقة (7):

$$
\vec{p}=\frac{(a-b) \vec{\nabla} b}{(\nabla b)^{2}+(a-b)^{2}}
$$

حيث: b تمثل شدة الكثافة في الصورة المرجعية في نقطة P و الصورة العائمة لنفس النقطة P. 
وتكمن مشكلة هذه الطريقة في حساسيتها للوضع الأولي للشبكة على

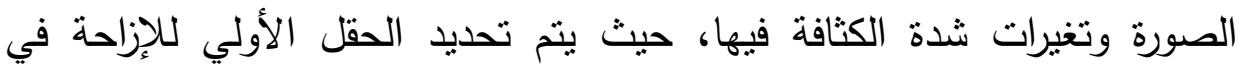

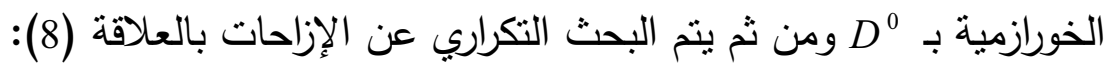

$$
p^{N}(x)=\frac{\left(a\left(x+p^{N-1}(x)-b(x)\right) \overrightarrow{\nabla b}\right.}{(\nabla b)^{2}+\left(a\left(x+\vec{p}^{N-1}(x)-b(x)\right)^{2}\right.}
$$

ب- الطريقة الهتهدة على العناصر الدنتهية Finite Elements Method

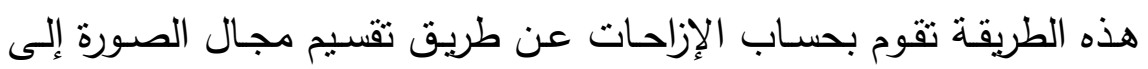

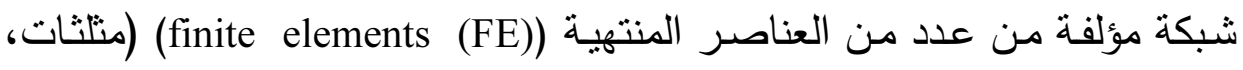

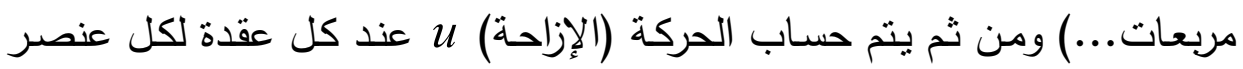

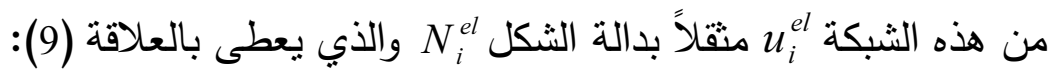

$$
u(x)=\sum_{i=1}^{3} N^{e l} u_{i}^{e l}(x)
$$

فالدالة الم في العقدة i للعنصر lel مع 3....

$$
N_{i}^{e l}=\frac{1}{2 S^{e l}}\left(a_{1}^{e l}+b_{1}^{e l} x+c_{1}^{e l} y+d_{1}^{e l} z\right)
$$

يتم إيجاد القيمة الدنيا للقوة الكامنة E من أجل كل عقدة i لفضاء عناصر الصورة

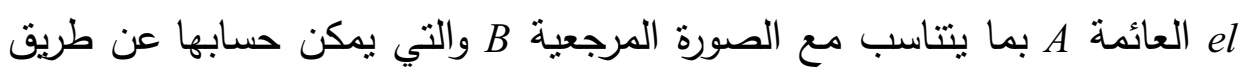

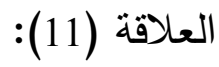

$$
E\left(u_{i}^{e l}\right)=\int_{\Omega} \sum_{j=1}^{3} u_{i}^{e l^{T}} B_{i}^{e l^{T}} D B_{j}^{e l} u_{j}^{e l} d \Omega+\mathrm{F} N_{i}^{e l} u_{i}^{e l} d \Omega
$$

حيث $\Omega$ تمثل مجال الدراسة، F تمتل متجه القوى المطبقة على الجسم المرن

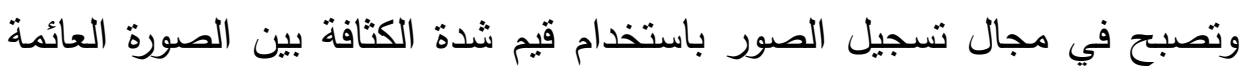

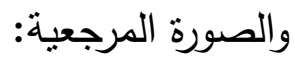




$$
\int_{\Omega}((A(x)+u(x))-B(x))^{2} d \Omega
$$

و D تمثل مصفوفة المرونة التي تميز خصائص المواد وu متجه الإزاحات و B متجه التشوهات. نجد القيمة الدنيا لهذا الدالة بحل المعادلة (13) :

$$
\begin{gathered}
\frac{d E\left(u_{i}^{e l}\right)}{d u_{i}^{e l}}=0 \\
\int_{\Omega} \sum_{j=1}^{3} B_{i}^{e l^{T}} D B_{j}^{e l} u_{j}^{e l} d \Omega=-\int_{\Omega} \sum_{j=1}^{3} \mathrm{FN}_{i}^{e l} d \Omega
\end{gathered}
$$

فتصبح معادلة القوة (11) كما يلي:

يمكن كتابة هذه المعادلة (14) كنظام مصفوفات لكل عنصر من العناصر المنتهية

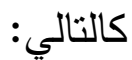

$$
\begin{gathered}
K^{e l} u^{e l}=F^{e l} \\
\text { حيث } K^{e l} \text { مصفوفة } 9 \text { * } 9^{\text {مع اعتبار أن }}{ }^{e l}=\int_{\Omega} B_{i}^{e l} D B_{j}^{e l} d \Omega
\end{gathered}
$$

و F متجه القوة يعرف بالعلاقة (17) في حالة تسجيل بين الصورة المرجعية والعائمة:

$$
F_{j}^{e l}=\int_{\Omega}(A-B) \nabla A N_{i}^{e l} d \Omega
$$

i,j تشير إلى زوج من العقد للعنصر el وتتفاوت بين • و r حيث تحسب ل لكل عنصر el g ومن ثم يتم تجميعهم في نظام شامل يشكل حقل التغييرات أي الحركة العامة أو القوة الثاملة للنظام. 


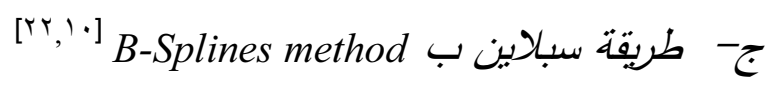
في هذه الطريقة يتت التعبير عن الإزاحات على افتراض أنها تتكون من حركتين إحداهما تمثل حركة محلية والثانية تمثل حركة شاملة ويتت التعبير عنهما على النحو التالي بالعلاقة (18):

$$
T(x, y, z)=T_{\text {Globale }}(x, y, z)+T_{\text {Locale }}(x, y, z)
$$

Tlobal $(x, y, z)$ عنها كما يلي:

$$
T_{\text {Globale }}(x, y)=\left(\begin{array}{ll}
\theta_{11} & \theta_{12} \\
\theta_{21} & \theta_{22}
\end{array}\right)\left(\begin{array}{l}
x \\
y
\end{array}\right)+\left(\begin{array}{l}
\theta_{13} \\
\theta_{23}
\end{array}\right)
$$

TLile $(x, y, z)$ الأشكال الحرة (Free Form Deformation (FFD): حيث يتم وضع شبكة منتظمة (20) (أبعادهـا

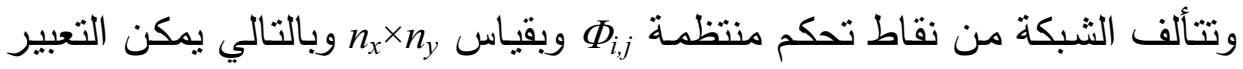

$$
\begin{gathered}
\text { عن هذا النوع من التحويلات بالعلاقة (21): } \\
\{\Omega=(x, y) \mid 0 \leq x \leq X, 0 \leq y \leq Y\} \\
T_{F F D}(x, y, t)=\sum_{l=0}^{3} \sum_{m=0}^{3} B_{l}(u) B_{m}(v) \phi_{i+l, j+m}
\end{gathered}
$$

حيث: عدد اللحظات المحصلة من مجموعة الصور الديناميكية وهي ، ب لحظة في حالة

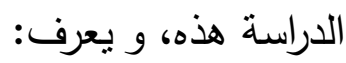

$$
i=\left\lfloor x / n_{x}\right\rfloor-1 ; j=\left\lfloor y / n_{y}\right\rfloor-1 ; u=x / n_{x}-\left\lfloor x / n_{x}\right\rfloor ; v=y / n_{y}-\left\lfloor y / n_{y}\right\rfloor
$$


و B تمثنل منحنيـات سبلاين ب المستخدمة في الخوارزميـة والتي هي من الدرجة الثالثة وذلك مـن أجل المحافظـة على استمرارية الأشكال وعدم حصسول انقطاع في الحركة المحتسبة وتعطى بمجموعة العلاقات (23) التالية:

$$
\begin{aligned}
& B_{0}(u)=(1-u)^{3} / 6 \\
& B_{1}(u)=\left(3 u^{3}-6 u^{2}+4\right) / 6 \\
& B_{2}(u)=\left(-3 u^{3}+3 u^{2}+3 u+1\right) / 6 \\
& B_{3}(u)=u^{3} / 6
\end{aligned}
$$

يبين الثكل ع توضع الثبكة الأولي على الصورة المرجعية في نهاية طور الانبسـاط، وتوضـع الشبكة على الصسورة في منتصف طور الانقباض أو الصورة العائمة والتي تمثل لحظة الانقباض الأعظمي للقلب.

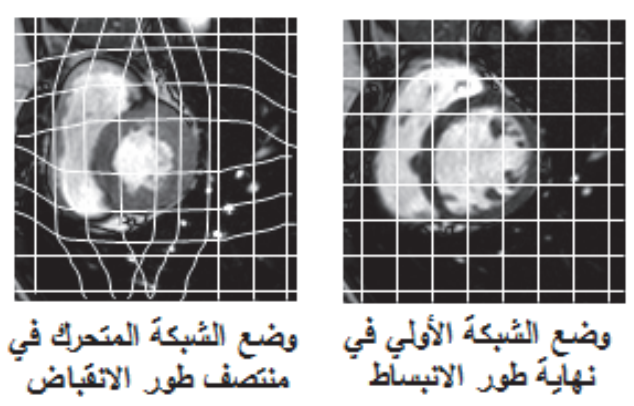

شكل عـ شبكة الأشكال الحرة المطبقة على الصورة المرجعية وإلعائمة.

r. التطبيق العملي: دراسة مقارنة على صور الرنين المغتطيسي القلبي

فيما يلي سيتم التعربف على المعطيات التي تم تطبيق وتجريب الخوارزميات الثلاث عليها من أجل تتبع حركة القلب والبارامترات المطبقة على كل خوارزمية ثم سيتم استعراض النتائج مع دراسة تحليلية لهذه النتائج. 


\section{ب - 1- المعطيات المحصلة}

مجموعة المعطيات المستخدمة لدراسـة المقارنة بين الطرق الثلاثة السابقة

هي عبارة عن مجموعة من الصور المحصلة بطريقة الرنين المغنطيسي (MRI)، لإنهان حيث تسمح هذه التقنية بتزويد صور للمكونات القلبية ذات دقة حيزية لهنية (spatial) عالية خـلال الدورة القلبية (CC) (cardiac cycle (التئي تعتبر مستوى التغيير

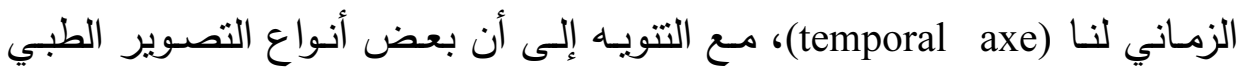

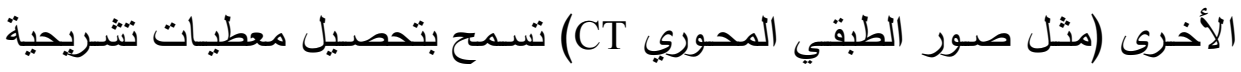

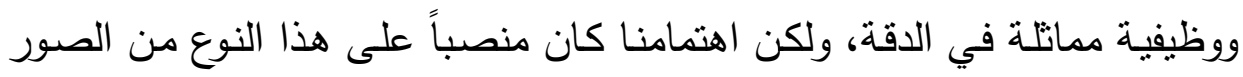
بالإضافة لتوافره لنا لإجراء هذا البحث.

تم تجميع الصور على متطوع سليم خالٍ من الآفات القلبية باستخدام جهاز رنين مغنطيسي 1 ا نيسلا وقد وقع الاختيار على تصوير مستوى قلبي نستطيع

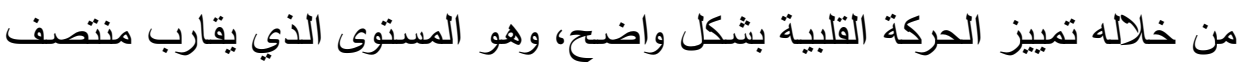
القلب باتجاه المحور الصغير (short axis) للقلب (انظر الثكل ه) وتتكون هذه

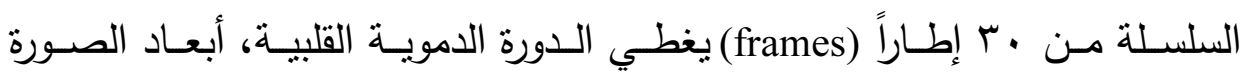
slice ) (الصـورة) (image dimentions)

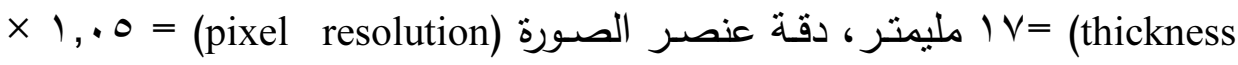

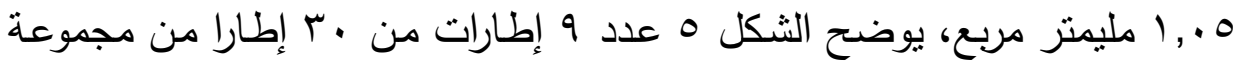

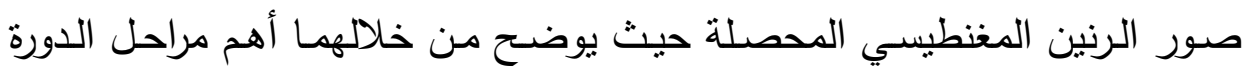
القلبية: بداية طور الانبساط (diastolic phase)، ومنتصف ونهاية طور الانقباض لرناض .(systolic phase) 


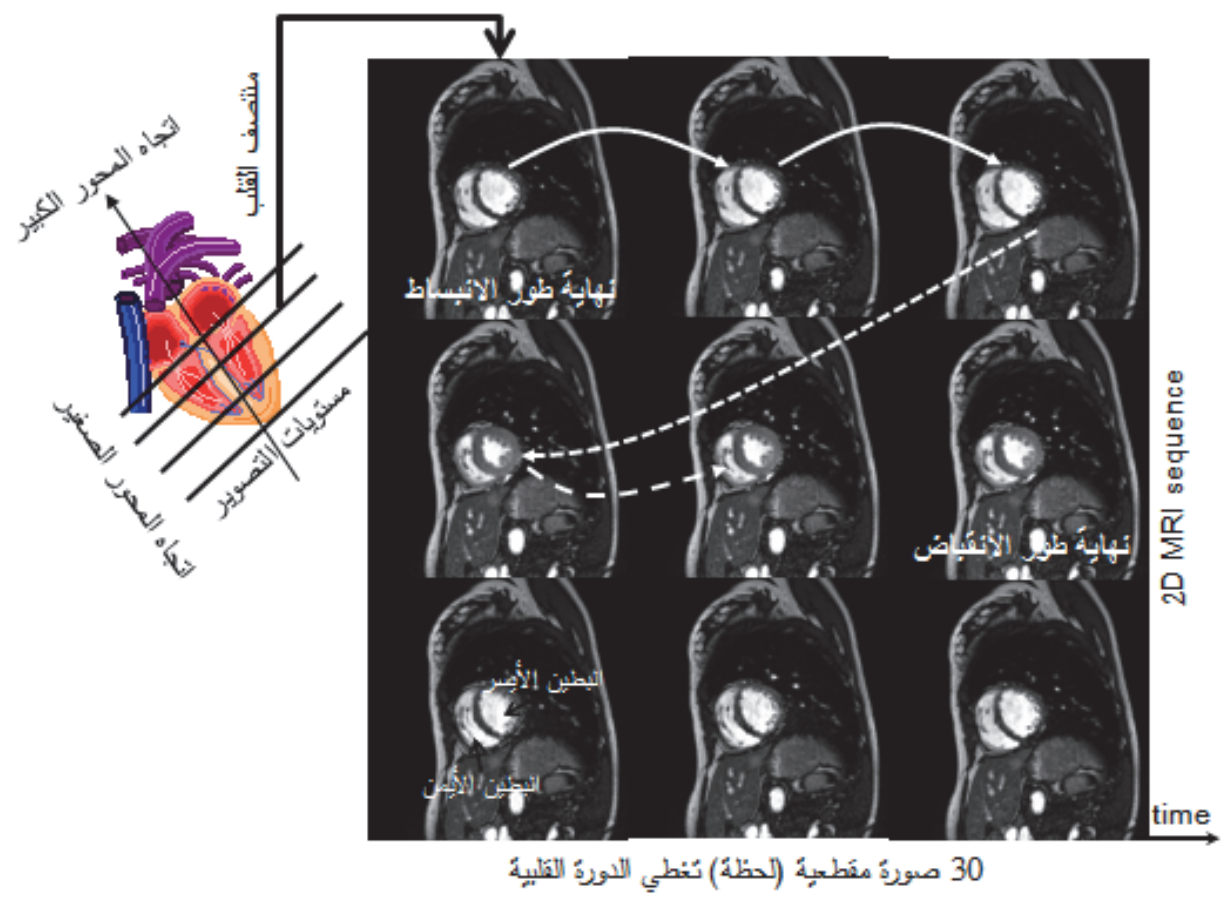

شكل ه . سلسلة صور الرنين المغتطيسي 2D الديناميكية.

r - بارامترات تطبيق الخوازميات على صور الزنبين المغنطيسي

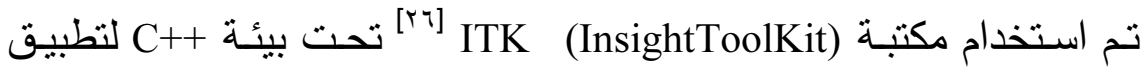
مختلف الخوارزميات التثلاث السابقة على مجموعة صور (MRI) القلبية السابقة. في البدايـة تم اختيار منطقة اهتمـام من الصسور وذلك بهدف تركيز العمل على الجزء القلبي فقط من الصور ، وبهدف تقليص فترة احتساب الحركة (الثكل ؟) ثم تم إعداد بـارامترات الخوارزميات الثلاثة لكل خوارزميـة على حدة بهدف تطبيقها على الصور القلبية وذللك لإتاحة عمل الخوارزميات على النحو الأمثل. وفيما يلي مختلف المعايير التي طبقت في هذا البحث: 


\section{بارمترات خواززمية ديمون}

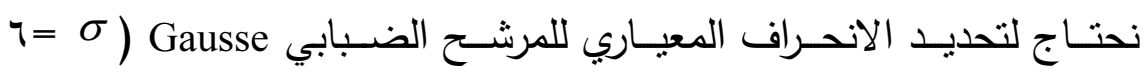

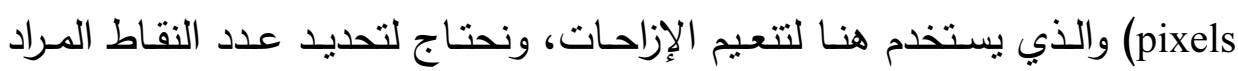

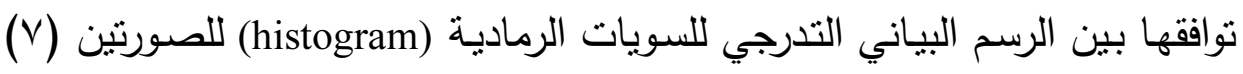
والحد الأقصى لعدد التكرارات ( . . (1) وهو لتحديد دالة الأمتلية لهذه الطريقة.

\section{بارمترات خوارزمية العناصر المنتهية}

نحتـاج لتحديد معيار التثـابه، وبالتجربـة وجدنا أن معيار النتـابه مجموع

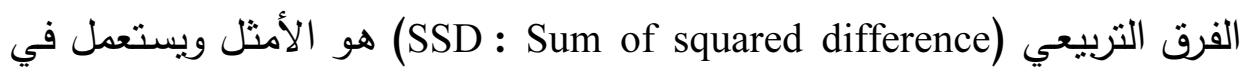
حالة وجود ضجيج ضبابي Gaussian ويعطى بالعلاقة (24):

$$
S S D=\frac{1}{N} \sum_{i \in \Omega_{A, B}^{T}}|A(i)-B(i)|^{2}
$$

وتم أيضـاً تحديــ الخطوة الزمنيـة وهـي تمثنل عدد التكرارات في إجرائيـة

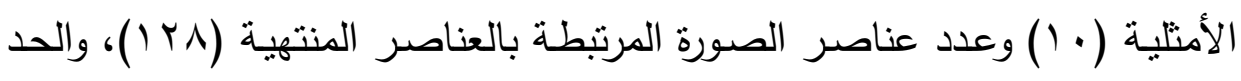

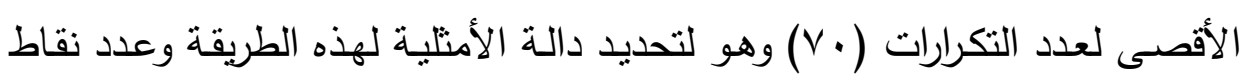

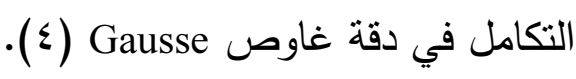
بارامترات خوارزمية سبالاين ب

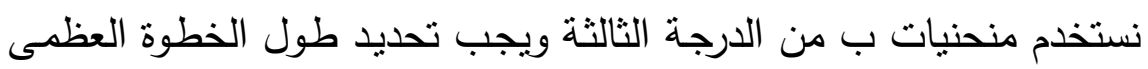
للمحسن وهي تمثل القيمة التي لا يجب تجاوزها في إجرائية التحسين (10) (pixels)،

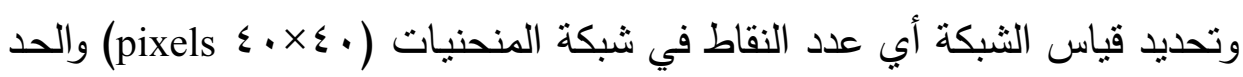
الأقصى لعدد التكرارات ( • (०). 


\section{ع ـ النتائج ومناقشتها}

بالنسبة للنتائج سنستعرض هنا مجموعة الصور للحظتين في طور الانبساط والتي تكون فيه الحركة مقاربة للصفر أو معدومة، ولصورتين في منتصف طور الانقباض حيث تكون حركة القلب عظمى. يبين الشكل 7 الطريقة المطبقة لتسجيل الصور القلبية من اختيار منطقة الاهتمام إلى تطبيق خوارزمية احتساب الحركة.

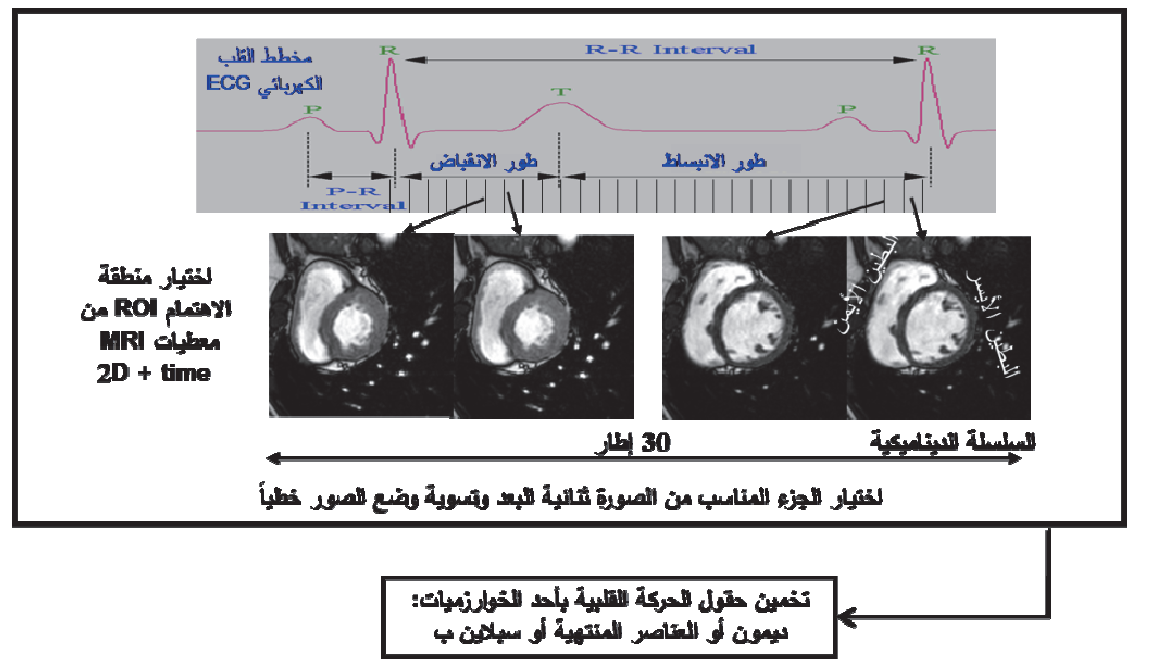

شكل 7 . طريقة تطبيق تسجيل الصور على المعيات المحصلة. فيما يلي سنقوم بإدراج النتائج العملية لكل خوارزمية على حدة (جداول (-ب): جدول ا. نتائج طريقة ديمون.

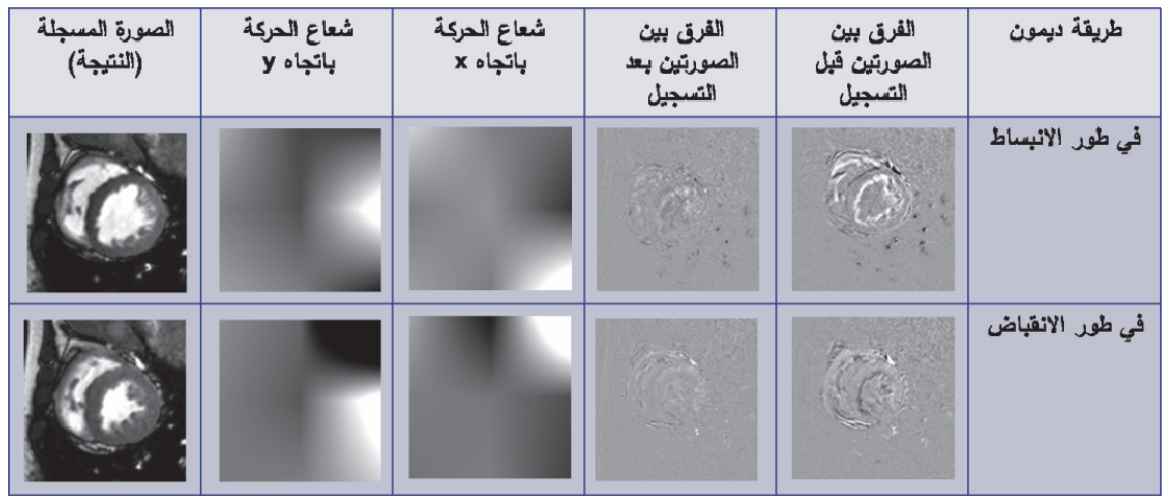


جدول r. نتائج طريقة العناصر المنتهية.

\begin{tabular}{|c|c|c|c|c|c|}
\hline الصورة المسجلة & شباتجاه الحركة & 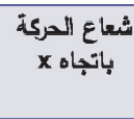 & الصفربتين بين & الصرقيتين قبل & طريقة العاصر \\
\hline Iro & & & & & في طور الآتبعاط \\
\hline $118=$ & & & & & في طود الانقباض \\
\hline
\end{tabular}

جدول r. نتائج طريقة سبلاين ب.

\begin{tabular}{|c|c|c|c|c|c|}
\hline الالصعرة المعبجة & بائجاه الحركة & بائجاع الحركة & الصوربتين بين & الصعربين قين & طريقة سبلاين ب \\
\hline & & & & & في طور الانبساط \\
\hline & & & & & في طود الاتقَاض \\
\hline
\end{tabular}

SSD يمثل جـدول ؛ التـالي قيم معيـار التشـابه مجموع الفـرق التربيعي للخوارزميات الثلاثة في لحظة التقلص الأعظمي. جدول ع ـ قيم مجموع الفرق التربيعي للخوارزميات الثلاثة.

\begin{tabular}{|c|c|c|}
\hline قيم SSD بعد عملية التسجيل & قيم SSD قبل عملية التسجيل & الطريقة \\
\hline 2982.16 & 2963.05 & ديمون \\
\hline 382902 & 2963.05 & العناصر الحرة \\
\hline 1903.16 & 2963.05 & سبلاين ب \\
\hline
\end{tabular}


يلاحظ مما سبق من النتائج: أن قيمة مجموع الفرق التربيعي SSD ينخفض أنما بشكل ملحوظ في طريقة سبلاين بالمقارنة مع القيمة قبل التسجيل وبعده. أما فيما يتعلق بطريقة العناصـر المنتهيـة فإن صـورة الفرق التي يتت حسـابها بعد عمليـة التسجيل فإن القوة المطبقة تقوم بتباعد الخوارزمية. وهذا واضح لأن قيمة مجموع الفرق التربيعي SSD بعد عملية التسجيل أعلى بكثير مما كان قبلها (الجدول؛). فمن الواضح أنه مع البيانات المتوافرة لدينا عملية تسجيل الصور بطربقة سبلاين ب تحقق أفضل النتائج. وعلى الرغم من الحصول على نتائج جيدة بطريقة ديمون لكنها ليست بجودة نتائج طريقة سبلاين. ونوضـح على الثكل V القيم المختلفة لمجموع الفرق التربيحي لهاتين الطربقتين. أما بالنسبة لطربقة العناصر المنتهية فلا بد من إيجاد إعدادات أفضل للحصول على نتائج أفضل لأنه لم يتضـح حتى الآن لماذا أعطت هذه النتائج السيئة على الصور الحقيقية.

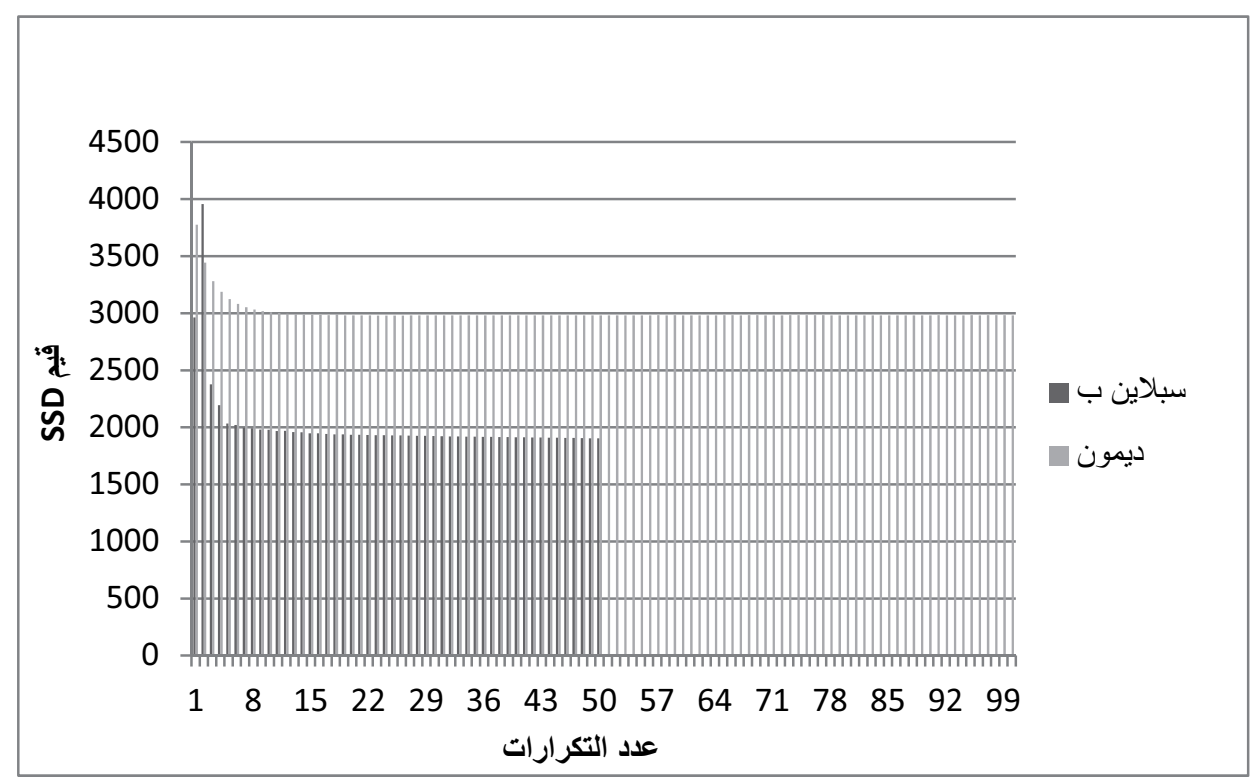

شكل V. القيم المختلفة لمجموع الفرق التربيعي لطريقتي سبلاين ب وديمون. 


\section{ه. الخلاصة والتوصيات}

قدم هذا البحث دراسـة شـاملة لطرق التسـيل غير الخطيـة بهدف تطبيقها

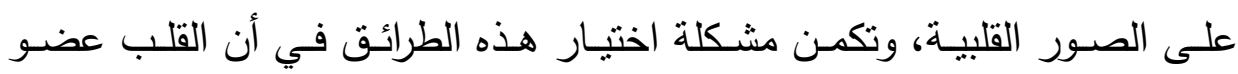

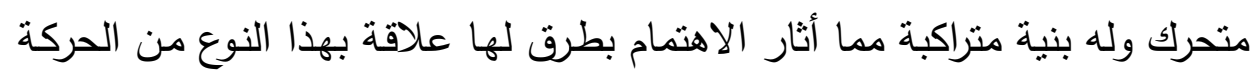
السريعة وذات الإزاحات الصغيرة (حركة القلب بسرعة أقل من ثانية واحدة / دورة

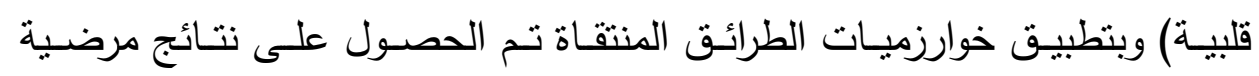
سمحت لنا بمقارنة هذه الأساليب.

إن هذه الدراسـة تحتاج للتطبيق في مجال أوسـع إمـا على نفس النوع من الصسور أو حتى على أنـواع أخرى منثل صسور الطبقي المحوري، بالإضـافة إلى إلى

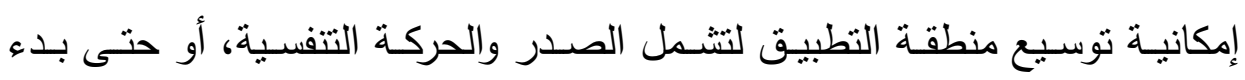
التطبيق ليثـمل الصـور ثثلاثية الأبعاد في حال توافرهـا. ومن خـلال هذه الدراسـة

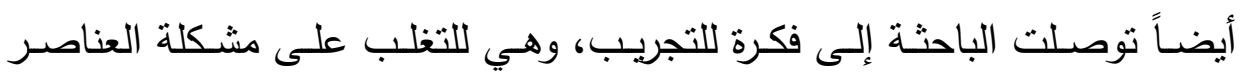
المنتهية التي واجهت تطبيق هذه الطريقة، وهي تقسيم منطقة الصورة إلى شبكات فرعية وهذا يسمح باستخدام حلول المعادلات التفاضلية الجزئية داخل المجال وفقاً

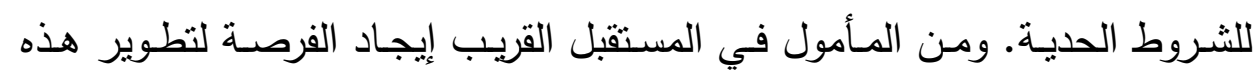
الخوارزمية.

\section{المراجع}

Behloul, F., Janier, M., Croisille, P., et al. "Automatic assessment of myocardial viability based on PET-MRI data fusion, Int. Conf. IEEE Engineering in Medicine and Biology Soc., pp: 429-495 (1998).

Hill, D. L. G., Batchelor, P. G., Holden, M. H. and Hawkes, D. J., Medical image registration, Phys. Med. Biol., 46 (1): 1-45 (2001).

Hajnal, J. V., Hill, D. L. G. and Hawkes, D. J., Medical Image Registration, Boca Raton, FL: CRC Press (2001).

Brown, L.G., A Survey of Image Registration Techniques, ACM Computer Survey, Vol. 24: 325-376 (1992).

Cerqueira, M.D., Weissman, N.J., Dilsizian, V., et al. Standardized myocardial 
segmentation and nomenclature for tomographic imaging of the heart: A statement for healthcare professionals from the cardiac imaging committee of the council on clinical cardiology of the American Heart Association (AHA). Circulation, 105(4): 539-542 (2002).

Planat, A. and Magnin, I.E., Estimation de mouvement par maillage actif avec prise en compte de discontinuities, Traitement du Signal, 18 (5-6): 383-401 (2001).

Sermesant, M., Clatz, O., Li, Z., Lanteri, S., et al., Parallel implementation of nonrigid registration using a volumetric biomechanical model. In: Second International Workshop on Biomedical Image Registration (WBIR), Philadelphia, PA, USA. LNCS 2717: 398-407 (2003).

Lancaster, J.L., Fox, P.T., Downs, H., Nickerson, D.S., Hander, T.A., El Mallah, M., et al. Global spatial normalization of human brain using convex hulls. Journal of Nuclear Medicine, 40: 942-55.

Diez, Y., Oliver, A., Llado, X. and Marti, R., Comparison of Registration Methods Using Mamographic Images, IEEE 17th International Conference on Image Processing (ICIP'10), pp: 4421-4424 ( 2010).

Rueckert, D., Sonoda, L.I., Hayes, C., et al., Nonrigid Registration Using Free-Form Deformations: Application to Breast MR Images, IEEE Transactions on Medical Imaging, 18 (8): 712-721 (1999).

Chandrashekara, R., Mohiaddin, R.H. and Rueckert, D., Analysis of myocardial motion in tagged MR images using nonrigid image registration, Proc. SPIE (Medical Imaging 2002: Image Processing): 1168-1179 (2002).

Rao, A., Sanchez-Ortiz, G.I., Chandrashekara, R., Lorenzo-Valdes, M., Mohiaddin, R. and Rueckert, D., Comparison of Cardiac Motion Across Subjects Using Non-rigid Registration, In: Medical Image Computing and Computer-Assisted Intervention (MICCAI'02). LNCS 2488: 722-729 (2002).

Smal, I., Carranza-Herrezuelo, N., Klein, S., Niessen, W.J. and Meijering, E.H.W., Quantitative comparison of tracking methods for motion analysis in tagged MRI. In IEEE international conference on Biomedical imaging (ISBI'11): 345-348 (2011).

Baker, S. and Matthews, I., Lucas-kanade 20 years on: A unifying framework: Part 1: The Quantity Approximated, the Warp Update Rule, and the Gradient Descent Approximation, International Journal of Computer Vision, 56 (3): 221-255 (2004).

Cebula, A.T., Gilland, D.R., Parker, J.G. and Chen, Y., Motion-based, multimodality image registration for cardiac imaging. In Proceedings of IEEE international conference on Biomedical Imaging: From Nano to Macro (ISBI'10): 508-511 (2010).

Pauna, N., Croisille, P., Costes, N., Reilhac, A., et al., A strategy to quantitatively evaluate MRI/PET cardiac rigid registration methods using a monte carlo simulator. In Functional Imaging and Modeling of the Heart (FIMH'03), LNCS 2674: 194-204 (2003).

Khurshid, K., McGough, R.J. and Berger, K. Automated cardiac motion compensation in PET/CT for accurate reconstruction of pet myocardial perfusion images. Phys Med Biol., 53: 5705-5718 (2008).

Ledesma-Carbayo, M.J., Kybic J., Desco, M., Santos, A., Suhling, M., Hunziker, P. and Unser, M., Spatio-temporal nonrigid registration for ultrasound cardiac motion estimation, IEEE Transactions on Medical Imaging, 24(9): 1113-1126 (2005).

Makela, T., Clarysse, P., Sipila, O., et al., A Review of Cardiac Image Registration Methods, IEEE Transactions on Medical Imaging, 21(9): 1011-1021 (2002). 
Lötjönen, J. and Mäkelä, T., Elastic Matching using a deformable sphere, In Medical Image Computing and Computer-Assisted Intervention (MICCAI'01): 541-548 (2001).

Declerc, J., Feldmar, J., Goris, M.L. and Betting, F., Automatic registration and alignment on a template of cardiac stress and rest reoriented SPECT Images, IEEE Transactions on Medical Imaging, 16: $727-737$ (1997).

Wierzbicki, M., Drangova, M., Guiraudon, G. and Peters, T.M. Mapping template heart models to patient data using image registration. In: Medical Image Computing and Computer-Assisted Intervention (MICCAI'04). LNCS 3217: 671-678 (2004).

Jasbir, Sra, Cardiac Image Registration: Rotational Error Correction and Gated Stabilization for Cardiac Motion, JAFIB, 2 (4): 856-869 (2011).

Thirion, J.P., Image matching as a diffusion process: an analogy with Maxwell's Demons, Medical Image Analysis, 2: 243-260 (1998).

Schnabel, J.A., Tanner, C.S., Degenhard, A., et al., Validation of nonrigid image registration using finite-element methods: application to breast MR images, IEEE Transactions on Medical Imaging, 22 (2003).

Yoo, T.S., Ackerman, M.J., Lorensen, W.E., et al. Engineering and Algorithm Design for an Image Processing API: A Technical Report on ITK - The Insight Toolkit. Proc. of Medicine Meets Virtual Reality, J. Westwood (ed.), IOS Press Amsterdam, pp:586-592 (2002). 


\title{
Tracking the Cardiac Motion in MRI by Using Nonlinear Image Registration
}

\author{
Rana Haddad \\ Department of Biomedical Engineering, Damascus University, Syria \\ haddadrana@yahoo.fr
}

\begin{abstract}
The process of tracking the heart motion helps the physicians to locate the actual incidence of myocardial disease like ischemia and on the possible risk degree of the affected area of the heart. In the present work, three different techniques of nonlinear image registration were implemented and applied to cardiac Magnetic Resonance Images (MRI) to track the heart motion, which is fast and small amplitude. The first of these techniques depends on Demons algorithm that is based on optical flow principle and the second approach based on applying control points by using the finite elements method to track the location change displacements of these points, the third technique depends on B-Splines curves and the displacements are calculated by Free Form Deformations (FFD) approach. The sum of squared difference (SSD) has been adopted as a similarity metric for the registration methods mentioned above to calculate the displacements of heart motion that is being used in cases of registering the mono-modality images and in case of Gaussian noise as in MR images. It has shown that the registration method based on B-Spline has given the best results and can be used efficiently for MR images registration and thus calculate the heart displacement.
\end{abstract}

Keywords: Magnetic resonance imaging, Medical image registration, Nonlinear registration methods, Heart motion tracking. 